\title{
Dynamic search for shortest multimodal paths in a transportation network
}

\author{
A. Di Febbraro, S. Sacone \\ Department of Communication, Computer, and System Sciences \\ University of Genova, Via Opera Pia 13, I-16145 Genova, Italy. \\ Tel: +39-10-3532983. Fax: +39-10-3532948. \\ e-mail: angela@dist.unige.it, simona@dist.unige.it
}

\begin{abstract}
Many transportation systems can be suitably represented as Discrete Event Systems. This is the case of the multimodal urban transportation network considered in this paper. In the simulation tool designed to reproduce its functioning, a module is included which allows to find the best multimodal paths between any pair of nodes in the network. The basic algorithm is an ad-hoc modified version of the Dijkstra algorithm to find the shortest paths in an oriented graph. Such an algorithm is complicated to include some choices left to users, regarding the number of paths required, the maximum time to travel, and the modes of transport to use. Then, a heuristic algorithm to find the best multimodal paths in a network integrating public and private modes of transport is proposed.
\end{abstract}

\section{Keywords}

Transportation, urban systems, Discrete Event Dynamic Systems.

\section{$1 \quad$ INTRODUCTION}

The integration between public and private transport services, with the aim of increasing the 'attractiveness' of public transportation means with respect to private ones, is a research topic of growing interest. Such an integration should lead to realize intermodality in transportation (Gedeon et al., 1993; Fernandez et al., 1994). With reference to the problem of integrating passenger transportation services in a urban area, the realization of efficient intermodal transportation systems should make people give up using their cars to move in the cities, which would result in both an optimization of the travelling times and a decrease in air pollution.

In this paper, a model of an intermodal transportation network which realizes the integration among urban public transportation services and private traffic is proposed. The system is represented as an oriented graph, in which the nodes are stations where it is possible to switch from a mode of transport to another one. In order to suit its peculiar characteristics, the considered transportation network is modelled as a Discrete Event Dynamic System (see, 
for instance, Cassandras, 1993). Due to the stochastic characteristics of such a system, some disturbances are also included. The behaviour of the discrete event system modelling the transportation network is studied by means of a special purpose designed simulation tool, which is the kernel of a urban traffic simulation program, designed to perform two major functions, almost independent of each other. The first objective is the validation of integrated timetables for the different transportation modes, so as to consider the various transportation services to be parts of a whole intermodal transportation system. In this sense, the traffic simulation program can evaluate the performance of the system, supporting the user in modifying adaptively a given timetable (Di Febbraro et al., 1994, 1995).

The second objective pursued is to give the users of the intermodal transportation network some real-time updated information about the state of the network itself. The optimal situation to realize is that in which a user can know, when approaching the transportation network, which path he had better follow to reach his destination, according to his requirements. Each user could enter the information system in the stations of the network, or even from his car. In particular, if a user approaches the network by car, he can evaluate where it is possible (and convenient) to leave his car and carry on with his journey using public modes of trasport. To this end, some intermodal stations have a parking area associated with.

This paper centres round the algorithms on which such an information system is founded. Two peculiar algorithms, resulting from the well-known Dijkstra algorithm (Christofides, 1975) and the method proposed by Yen (1971), are proposed to find the best multimodal paths, considering, respectively, only public modes of transport and both private and public modes of transport. Information made available to users regard essentially : i) the travelling times along all the possible paths, intermodal or not, between any pair of nodes in the network; ii) the availability of places in the parking areas associated with some macronodes; iii) the occurrence of irregular traffic conditions in some part of the network. Some examples of application of the algorithms proposed are presented.

\section{THE DISCRETE EVENT MODEL OF THE TRANSPORTATION NETWORK}

The multimodal transportation network is modelled as an oriented graph, whose fundamental elements are nodes, macronodes, and links. A node is a station for a single mode of transport, and it can only exist as a part of a macronode. A macronode is an intermodal station, that is, a place where people can change mode of transport, or simply enter the transportation system. Therefore, a macronode is composed of one or more nodes. A link is a unidirectional path which connects two macronodes and is devoted to a single transportation mode. There are special links, named inner links, which connect two nodes in a macronode and can be traversed only on foot.

It is assumed that users can approach the urban integrated transportation system using any means of transport. To model the arrivals of passengers by private means of transport, parking areas are considered to be located near some macronodes of the network, where passengers can leave their means of transport, if room is available. The existence of a parking area at a macronode and the availability of places in it play an important role in modelling the integration between public and private transport means. 
For the sake of simplicity, it is assumed that the considered transportation system consists of three different kinds of public transportation services : underground, railway, and bus. However, there is no difficulty in adding and/or changing modes of transport. In this framework, the links which connect the $\mathrm{N}$ macronodes in the network are divided into three classes: a) bus links (B-links); b) railway links $(R$-links); c) underground links (U-links). As shown in Figure 1, each macronode can have at most six links in common with another macronode.

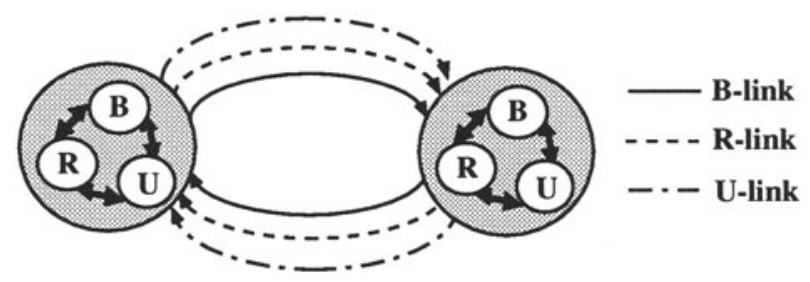

Figure 1 A generic pair of macronodes.

Macronodes $n, n=1, \ldots, N-1$, represent the real intermodal stations of the transportation system under study, while the last macronode, macronode $\mathbf{N}$, is a special macronode, i.e., a 'virtual' intermodal station which represents the outer world with respect to the network. In other words, each macronode which connects the urban area with its surroundings has a link towards macronode $\mathbf{N}$. The presence of such a virtual macronode allows to model the considered transportation system as a closed network. This yields a major advantage consisting in the possibility of applying particular storage methods for closed graphs, which reduce sensibly the computational load. Moreover, the presence of macronode $\mathrm{N}$ also allows to model the movement of those means of transport which go, or come from outside the considered urban transportation network.

The entities of the model, i.e., its components requiring explicit representation (Banks and Carson, 1984), are : macronodes, nodes, links, and transportation means. With each class of entities, static quantities (parameters) and dynamic quantities (state variables) are associated. The discrete events that describe the evolution of the considered urban transportation network, and then rule the functioning of the traffic simulation tool, are divided into two classes (Di Febbraro et al., 1995):

1. events that describe the nominal traffic conditions;

2. events that represent unpredictable conditions affecting the system.

The evolution of the system is reproduced by means of discrete event simulation, which can be viewed as the modelling over time of a system in which changes in the state only occur at discrete time instants, i.e., those instants when events occur. Each type of event is associated with an operational procedure which, once invoked, modifies the state variables of the model in dependence of the event occurred. Such procedure also schedules the events which will occur as a consequence of the current event, and cancels those events which the current event prevents from occurring. 


\section{HEURISTIC SEARCH FOR THE BEST INTERMODAL PATHS}

To find the best intermodal paths between a pair of nodes, two different algorithms are proposed. The very basic algorithm is an ad-hoc modified version of the well-known Dijkstra algorithm to find the shortest paths in an oriented graph (Christofides, 1975). Before describing in detail the proposed algorithm, it is worth introducing some notation. The transportation network is modelled as an oriented graph $G=\{S, L\}$, where $S$ is the set of the macronodes of the network, $\operatorname{card}(S)=\mathrm{N}_{\mathrm{s}}$, and $\mathrm{L}$ is the set of links of the network, $\operatorname{card}(\mathrm{L})=\mathrm{N}_{\mathrm{l}}$. The origin macronode of a link is identified by the function $O: \mathrm{L} \rightarrow \mathrm{S}$, such that $O(1)=\mathrm{s}, 1 \in \mathrm{L}, \mathrm{s} \in \mathrm{S}$, means that macronode $\mathrm{s}$ is the origin macronode of link 1 . In an analogous way, function $D: \mathrm{L} \rightarrow \mathrm{S}$ identifies the destination macronode of a link, i.e., $D(\mathrm{l})$ represents the destination macronode of link 1 . As described above, the links are classified in dependence of the mode of transport to which they are devoted; let $M$ be the set of the modes of transport present in the considered network and $\operatorname{card}(\mathrm{M})=\mathrm{N}_{\mathrm{m}}$. In the considered case, $\mathrm{N}_{\mathrm{m}}=4$ and $\mathrm{M}=\{\mathrm{B}, \mathrm{R}, \mathrm{U}, \mathrm{C}\}$, where $\mathrm{B}, \mathrm{R}, \mathrm{U}$, and $\mathrm{C}$ stand for bus, railway, underground, and cars, respectively. Moreover, let $M: \mathrm{L} \rightarrow \mathrm{M}$ be the function such that $M(\mathrm{l})=\mathrm{m}, 1 \in \mathrm{L}, \mathrm{m} \in \mathrm{M}$ means that link $I$ is devoted to mode of transport $\mathrm{m}$.

Using the variables introduced so far, the topology of graph $\mathrm{G}$ is defined by means of an adjacency matrix $A, \operatorname{dim}(A)=N_{s} \times N_{s} \times N_{m}$, whose elements are:

$\mathrm{a}(\mathrm{i}, \mathrm{j}, \mathrm{m})= \begin{cases}1 & \text { if } \exists 1 \in \mathrm{L}: O(1)=\mathrm{i}, D(1)=\mathrm{j}, M(1)=\mathrm{m} \\ 0 & \text { otherwise }\end{cases}$

$\forall i, j \in S, m \in M$

Other matrices represent the travel times on the links and the waiting times in the nodes. Let $T, \operatorname{dim}(\mathbf{T})=\mathrm{N}_{\mathrm{s}} \times \mathrm{N}_{\mathrm{s}} \times \mathrm{N}_{\mathrm{m}}$, be the matrix containing the time-varying travel times on the links; if $\tau_{i, j}, i, j \in S$, denotes the present travel time on link $1,1 \in \mathrm{L}, O(1)=\mathrm{i}, D(1)=\mathrm{j}$, the generic element of $\mathbf{T}$ can be written as:

$t(i, j, m)= \begin{cases}\tau_{i, j} & \text { if } a(i, j, m)=1 \\ +\infty & \text { otherwise }\end{cases}$

$\forall \mathrm{i}, \mathrm{j} \in \mathrm{S}, \mathrm{m} \in \mathrm{M}$

Then, let IL denote a matrix, $\operatorname{dim}(\mathbf{I L})=\mathrm{N}_{\mathrm{s}} \times \mathrm{N}_{\mathrm{m}} \times \mathrm{N}_{\mathrm{m}}$, whose generic element $\mathrm{il}(\mathrm{i}, \mathrm{m}, \mathrm{n}), \mathbf{i} \in \mathrm{S}$, $m, n \in M$, represents the travel time on the inner link within macronode $i$, and connecting the node (station) of mode $m$ with the node (station) of mode $n$. Of course, il $(i, m, m)=0$. The generic element of matrix $\mathbf{W T}, \operatorname{dim}(\mathbf{W T})=\mathrm{N}_{\mathrm{S}} \times \mathrm{N}_{\mathrm{m}}$, namely $w t(\mathrm{i}, \mathrm{m}), \mathrm{i} \in \mathrm{S}, \mathrm{m} \in \mathrm{M}$ represents the time to wait presently in macronode $i$ before a means of transport of mode $m$ arrives. Last, to model the time-varying availability of places in the parking areas associated with the nodes, a vector $\mathbf{p}, \operatorname{dim}(\mathbf{p})=\mathrm{N}_{\mathrm{s}}$, is introduced, and its generic element is defined as

$\mathrm{p}(\mathrm{i})= \begin{cases}0 & \text { if there is no place in the parking area at node } \mathrm{i} \\ 1 & \text { if there is at least a place in the parking area at node } i \\ +\infty & \text { if there is no parking area associated with node } i\end{cases}$

$\forall i \in S$

If macronode $i$ is associated with a parking area, the value of $p(i)$ is randomly determined by taking into account the probability of finding free places in it. Such a probability varies in dependence of macronode $i$ and of the time of the day. It is worth noting that only matrices $\mathbf{A}$ 
and $I L$ have constant elements. Matrices $\mathbf{T}$ and $\mathbf{W T}$ are updated in real time based on the data provided by the simulation kernel, whereas the components of vector $\mathbf{p}$ are stochastic variables.

As said above, the algorithm which solves the problem of finding the shortest path from an origin macronode $o$ to a destination macronode $d, o, d \in S$, considering only public means of transport, is derived from the Dijkstra algorithm. The main difference between the classical version of Dijkstra algorithm and the proposed one stands in the cost function associated with each link. Here, such a cost not only depends on the origin and destination nodes of the link, but also on the mode of transport currently used. Then, the cost associated with link $1,1 \in \mathrm{L}$, can be expressed by a function $c(i, j, m)$, where $i=O(l), j=D(l)$, and $m \in M \backslash\{C\}$ is the mode of transport used to reach node $i$, taking on the form:

$$
\mathrm{c}(\mathrm{i}, \mathrm{j}, \mathrm{m})=\min _{\mathrm{n} \in \mathrm{M} \backslash\{\mathrm{C}\}}\{\mathrm{t}(\mathrm{i}, \mathrm{j}, \mathrm{n})+\mathrm{il}(\mathrm{i}, \mathrm{m}, \mathrm{n})+\mathrm{wt}(\mathrm{i}, \mathrm{n})\}
$$

The basic version of the algorithm has been complicated to include some further possibilities offered to users, who can choose about:

1. the number $k, k \geq 1$, of best paths to find between the indicated pair of nodes;

2. the maximum travel time $t_{\max }$ of the path from node o to node $d$, i.e., a user can fix the maximum time he is willing to spend to reach his destination;

3. the modes of transport utilized, i.e., a user can choose which modes he prefers to use to reach his destination.

It is apparent that the introduction of the above possibilities for the user results in additional constraints to impose on the solution of the optimization problem. If only point 1 is specified, the problem to solve is that of finding the $\mathrm{k}$ best paths to move in the network from node o to node $\mathrm{d}$. We solve such a problem by applying the method proposed by Yen (1971), based on the modified version of Dijkstra algorithm that we have proposed above. Almost the same holds if also the maximum travel time is specified. In fact, the structure of the problem to solve is left essentially untouched with respect to the previous case. Only an additional constraint on the travel time has to be taken into account, so that only those paths with overall travel times less than $t_{\max }$, if any, should appear in the list of paths given as an output.

As for decisional aspect 3 , the possibility for the users of neglecting some mode of transport does not lead to a trivial complication of the problem. Actually, if a user chooses to eliminate one or more modes of transport from his path, the resulting graph can become not connected. If so, we would not be able to solve the problem using the algorithm proposed so far, as the Dijkstra algorithm only applies to connected graphs. To deal with this aspect, a special procedure has been implemented to check the type of structure of the graph and act consequently. If the graph does not turn out to be connected, such a procedure removes from it the isolated macronodes. Once a connected topology has been reached again, the algorithm to apply is the same as above, the only difference standing in the fact that the set of possible modes of transport to consider is now only a subset of $M$.

The algorithms described so far only work on the public transportation network, without taking specifically into account the presence of private traffic. Being the primary aim of our work the integration of the public transportation services with the private traffic, we state now a heuristic algorithm which serves this purpose. It is assumed that this algorithm is run to answer a question posed by a user approaching a macronode of the network travelling by car. In fact, the algorithm searches for the best multimodal paths in which the first part (possibly 
the whole path) has to be covered by car, if any such a path exists, according to the following steps.

\section{Algorithm}

Given an origin macronode 0 and a destination macronode $d$ :

1. Define the set of nodes $S^{\prime}=\{s \in S, s \neq 0$ and $p(s)=1\}$;

2. $\forall s \in S^{\prime}$, find, by applying the Dijkstra algorithm on graph $G^{\prime}=\left\{S, L^{\prime}\right\}$, where $L^{\prime}$ is the set of the links relevant to mode $C$, the shortest path from macronode o to macronode $s$ and, then, define vector $t_{c}, \operatorname{dim}\left(t_{c}\right)=\operatorname{card}\left(S^{\prime}\right)$, such that $t_{c}(s), s \in S^{\prime}$, is the travel time on the path determined;

3. $\forall s \in S^{\prime}$, find, by applying the modified version of the Dijkstra algorithm described above, the shortest multimodal path from $s$ to $d$; then, define vector $t_{\mathrm{mm}}, \operatorname{dim}\left(t_{\mathrm{mm}}\right)=$ card( $\left.S^{\prime}\right)$, such that $t_{\mathrm{mm}}(\mathrm{s}), \mathrm{s} \in \mathrm{S}^{\prime}$, is the travel time of such a path;

4. Determine the best path to move from o to $d$, by choosing macronode $i \in S^{\prime}$, such that:

$$
\mathbf{t}_{\mathrm{c}}(\mathrm{i})+\mathbf{t}_{\mathrm{mm}}(\mathrm{i})=\min _{\mathrm{s} \in \mathrm{S}^{\prime}}\left\{\mathbf{t}_{\mathrm{c}}(\mathrm{s})+\mathbf{t}_{\mathrm{mm}}(\mathrm{s})\right\}
$$

The above algorithm can be easily combined with the three additional possibilities offered to users described above.

\section{SIMULATION RESULTS}

In this section, some experimental results showing the effectiveness of the heuristic algorithm proposed in the previous section are presented and discussed. The considered case study is relevant to the transportation network. Such a network is composed of 80 links and 21 macronodes, each of which consists of at least two nodes. In particular, in the network there are 21 bus stations, 13 railway stations, and 11 underground stations. In order to evaluate its behaviour with reference to the case study, the algorithm, which has been implemented in $\mathrm{C}$ programming language, has been run with many different input requests. Two significant examples are reported hereafter. The first example consists in a request to find the four best paths to move from macronode 6 to macronode 19 using private and/or public means of transport. The output of the algorithm is shown in Table 1, in which, for each path found, the following data are reported:

1. the total travel time (TTT) in seconds;

2. the sequence of the macronodes which compose the path (Paths); the nodes where the mode of transport changes are followed by the indication of the new mode adopted;

3. the number of macronodes $(N M)$ in the path;

4. the number of mode changes $(N M C)$ in the path. 
Table 1

\begin{tabular}{lllll}
\hline & $T T T(\mathrm{sec})$ & Paths & NM & NMC \\
\hline 1 & 900 & 6-R-3-5-8-12-U-15-R-19 & 7 & 2 \\
2 & 960 & 6-C-9-U-8-12-15-R-19 & 6 & 2 \\
3 & 960 & 6-B-9-U-8-12-15-R-19 & 6 & 2 \\
4 & 1020 & 6-R-3-5-8-U-12-15-B-19 & 7 & 2 \\
\hline
\end{tabular}

As a second example, we report the case in which the three best paths to move from macronode 2 to macronode 20, with a maximum travel time of 800 seconds and by using only public transportation services, have been asked to the program. The solution is shown in the following table.

Table 2

\begin{tabular}{lllll}
\hline & TTT(sec) & Paths & NM & NMC \\
\hline 1 & 720 & 2-B-5-R-8-U-12-15-20 & 6 & 2 \\
2 & 780 & 2-B-4-U-8-12-15-20 & 6 & 1 \\
3 & 780 & 2-B-5-R-8-12-U-15-20 & 6 & 2 \\
\hline
\end{tabular}

It is worth noting the importance of the information about the number of changes of modes of transport to be made along a path. In this simple case, the user would probably choose the second path found, because it presents a total travel time very close to the optimum one, and a number of changes of modes less than the one requested by the best path determined.

\section{CONCLUSIONS}

The problems related to the integration of public and private means of transport in urban areas have been dealt with in this paper. The resulting intermodal transportation network has been modelled as a discrete event system, whose behaviour has been studied by means of a special-purpose discrete event simulator. Such a simulator includes two major modules: a traffic simulation kernel and a passenger information service, which perform its main functions.

In particular, the paper was focused on the functioning of the on-line information service included in the simulator, which is able to provide updated information about the best intermodal paths to reach any destination in the network. Such best paths are time-varying as they follow the system dynamics, i.e., the traffic conditions. The heuristic algorithm used to find the best paths, including not only public modes of transport but also private ones, is based on the Dijkstra algorithm and the method proposed by Yen. Examples of application of 
this special algorithm to find the best multimodal paths have been presented and commented on.

The usefulness of the proposed approach can be improved. In fact, whenever the passenger information system receives a request for a best multimodal path from a user, it provides this path based only on the present state of the transportation network. Actually, this yields good results only if the network is not too big, and the traffic has slow dynamics. Work is in progress to extend the proper applicability of the proposed heuristic algorithm by finding a good technique to estimate the future values of the variables under concern.

\section{REFERENCES}

Banks, J., and J.S. Carson (1984) Discrete-Event System Simulation. Prentice-Hall, Englewoods Cliffs, NJ.

Cassandras, C. G. (1993) Discrete Event Systems. Irwin and Aksen Ass., Boston, MA.

Christofides, N. (1975) Graph Theory. Academic Press, London, UK.

Di Febbraro, A., V. Recagno, and S. Sacone (1994) Discrete Event Simulation of an Intermodal Transportation System. Proc. 1994 European Simulation Multiconference, Barcelona, Spain 1100-1104.

Di Febbraro, A., V. Recagno, and S. Sacone (1995) INTRANET : a new Simulation Tool for Intermodal Transportation Systems, to appear in Simulation Practice and Theory.

Fernandez, E., J. De Cea, M. Florian, and E. Cabrera (1994) Network equilibrium models with combined modes, Transportation Science 28, 182-192.

Gedeon, C., M. Florian, and T.G. Crainic (1993) Determining origin-destination matrices and optimal multiproduct flows for freight transportation over multimodal networks, Transportation Research, Part B 27B, 351-368.

Yen, J.Y. (1971) Finding the k-shortest, loopless paths in a network. Man. Sci., 17, 712. 